\title{
PARACOCCCIDIOIDOMICOSE COM MANIFESTAÇÃO BUCAL - CASUÍSTICA EM VOLTA REDONDA-RJ NOS ANOS DE 2005 A 2008
}

\section{PARACOCCIDIOIDOMYCOSIS WITH ORAL EXPRESSION - MATERIAL VOLTA REDONDA-RJ IN YEAR 2005 A 2008}

\author{
Oriental Luiz de NORONHA FILHO ${ }^{1}$ \\ Claudio VALENTE ${ }^{2}$ \\ ${ }^{1}$ Cirurgião-Dentista, Especialista em Saúde da Família, Especialista em Gestão Clínica na Atenção \\ Primária, Especialista em Cirurgia e Traumatologia Bucomaxilofaciais, Especializando em Radiologia e \\ Imaginologia Odontológica, Mestre em Saúde Coletiva e Cirurgião-Dentista da ESF Olaria - Município \\ de Cruzília - MG. \\ ${ }^{2}$ Cirurgião-Dentista, Professor e Coordenador do Curso de Especialização em Cirurgia e Traumatologia \\ Bucomaxilofaciais do Unifoa, Especialista e Mestre em Cirurgia e Traumatologia Bucomaxilofaciais e \\ Doutor em Estomatologia.
}

RESUMO: A Paracoccidioidomicose, antigamente conhecida como Blastomicose Sul Americana, pode ser instalada pela via inalatória ou por lesões na pele ou nas mucosas. Má higiene e doenças bucais são as portas de entrada mais comuns para este fungo, que uma vez na corrente sanguínea se torna sistêmica por infectar pulmões, baço, fígado, rins, pâncreas, ossos e linfonodos. O fungo se desenvolve em vegetais e solos férteis em áreas geográficas e climáticas especificas comuns as regiões do vale do rio Paraíba do Sul. A doença tem preferências pelo gênero masculino, pela raça branca e pela faixa etária entre 41 e 50 anos. A lesão primaria bucal compromete a língua, a gengiva, os lábios e demais mucosas bucais, de forma isolada ou associada. As lesões bucais apresentam-se como lesões bem localizadas e volumosas com aspecto de microgranulações, assemelhando-se a amora. O presente estudo avaliou prontuários médicos da Secretaria Municipal de Saúde de Volta Redonda, cuja lesão primaria tenha sido na boca. Foram selecionados 11 prontuários que corroboraram com os dados literários, enfatizando a língua como a mais acometida e a associação com a má-higiene bucal e hábitos orais como tabagismo e etilismo. Todos avaliados apresentavam infecção pulmonar e foram tratados clinicamente através da administração de sulfas, em regime ambulatorial e por período de seis a doze meses. Concluiu-se poder ser esta região endêmica e a importância do papel do Cirurgião Dentista no diagnostico precoce da doença.

Palavras Chave: Paracoccidioidomicose, Blastomicose, Epidemiologia, Incidência e Diagnóstico.

ABSTRACT: Paracoccidioidomycosis, formerly known as South American Blastomycosis, can be installed by inhalation or by skin lesions or mucous membranes. Poor hygiene and oral diseases are the ports of entry most common for this fungus, once in the bloodstream becomes systemic to infect the lungs, spleen, liver, kidneys, pancreas, bones and lymph nodes. The fungus grows on plants and soils in 
geographical and climatic conditions common areas of the valley of the Paraíba do Sul. The disease has preferences for males, for white and aged between 41 and 50. The primary lesion compromises the oral tongue, gums, lips and other mucous, isolated or associated. The oral lesions appear as lesions located and bulky appearance of micro granules, resembling a blackberry. This study examined medical records of the Municipal Health Department of Volta Redonda, whose primary lesion was in the mouth. We selected 11 patient records that corroborated with the literary data, emphasizing the language as the most affected and the association with bad oral hygiene and oral habits such as smoking and alcohol consumption. All evaluated had pulmonary infection and were treated clinically by the administration of sulfa drugs, outpatient and six to twelve months. It was concluded this could be an endemic area and the importance of the role of the Dental Surgeon in the diagnosis of the disease.

Key words: Paracoccidioidomycosis, Blastomycosis, Epidemiology, Incidence and Diagnosis.

\section{Autor Correspondente:}

Oriental Luiz de Noronha Filho

Rua Ângela Maria Sciane, 231- Vila Magalhães - Cruzília (MG) - 37445000

orientalnfilho@gmail.com

\section{INTRODUÇÃO}

A Paracoccidioidomicose é uma infecção fúngica profunda causada pelo Paracoccidioides Brasiliensis, que tem por predileção o gênero masculino e pela raça de pele branca, de pacientes oriundos das Regiões Sul, Sudeste e Centro-Oeste do Brasil, principalmente aqueles que trabalham ou exercem alguma função na atividade rural.

De acordo com Cerri et al., (1986), o fungo tem como habitat os vegetais e a terra. Incide normalmente em ares de solo fértil de vales, nas proximidades de grandes rios e vegetações abundantes ou extensas lavouras, acometendo principalmente trabalhadores rurais. Portanto os principais fatores climáticos e geográficos presentes nas áreas endêmicas da micose são: temperatura entre 11 e $30^{\circ} \mathrm{C}$; pluviometria entre 800 e $2.000 \mathrm{~mm}$ por ano; altitude entre 100 e 1.500 metros; matas tropicais e subtropicais e solos ácidos. (CABRAL et al., 2000; LONDERO, 1987). Estas características climáticas, pluviométricas e vegetativas assemelham-se com a região de Volta Redonda, município onde foi realizada a presente pesquisa. (IBGE, 2000).

A transmissão do fungo é feita por via inalatória ou através da contaminação pela pele ou mucosas 
lesadas. No que se refere à via de penetração do fungo no organismo humano, sugere a inalação de esporos do fungo e a lesão primária pulmonar como origem da infecção; entretanto, não descarta a possibilidade do Paracoccidioides Brasiliensis instalarse no hospedeiro através de via cutânea ou mucosa devido a lesões primárias decorrentes de traumas lacerantes ou lesões bucais (CABRAL et al,. 1994; LONDERO et al., 1987). Podem ocorrer transmissões por outras vias de penetração do fungo, a exemplo da mucosa anal pela higienização, após a defecação, com folhas de vegetação rasteira. A partir daí, o fungo pode caminhar e se instalar no intestino (PONTES et al., 2000; BORAKS, 1996).

Esta infecção fúngica manifestase como comprometimento cutaneomatoso, ganglionar e visceral, em que se destacam pulmão, fígado, baço, pâncreas, rim, supra-renal e osso. $\mathrm{O}$ acometimento do sistema nervoso central (SNC) é pouco freqüente, variando nas diversas casuísticas entre $9,6 \%$ a $12,5 \%$, predominando no encéfalo. As lesões podem apresentar-se tanto na forma meníngea como pseudotumoral (granulomatosa) ou mista. $\mathrm{O}$ acometimento intramedular é considerado raro, com apenas três casos registrados na literatura até o momento, nenhum associado à lesão cerebral como foi relatado (MORATOFERNANDEZ et al., 1991). O envolvimento pulmonar crônico está presente em cerca de $70 \%$ dos pacientes, os quais exibem tosse, dispnéia, febre, perda de peso e hemoptise (ORTEGA et al,. 1996). Na maioria dos casos ocorrem lesões primárias em terço médio de pulmões que se disseminam através da corrente sangüínea para os gânglios linfáticos e tecidos originando lesões secundárias.

$\mathrm{Na}$ cavidade bucal, as lesões primárias da paracoccidioidomicose localizam-se nos tecidos periodontais e mucosas. Nas mucosas surge freqüentemente como uma lesão ulcerada, de evolução lenta, com aspecto granulomatoso e avermelhado, semelhante à superfície de uma amora, denominada de estomatite moriforme. A Sialorréia e a sensação de prurido, dor e ardor também são encontradas, sendo que nos lábios a lesão provoca tumefação difusa (BISINELI et al., 1998).

As lesões iniciais têm como preferência marcante a cavidade bucal, sendo esta uma das suas características. Esta predileção em grande parte atinge a gengiva marginal e a papilar, entretanto 
ela pode iniciar-se pelas amídalas e pela mucosa labial. (CERRI et al., 1986; LASCALA, MOUSSALI, 1983).

As lesões bucais são geralmente acompanhadas de linfadenopatia submandibular ecervical, que inicialmente apresentam-se duras e dolorosas e se tornam, com o tempo, amolecidos e aderidos à pele, onde podem fistulizar, liberando uma exsudação rica em proteínas (PONTES et al., 2000). A principal característica da lesão na mucosa é a presença de micropápulas avermelhadas, puntiformes, assentadas em meio à membrana esbranquiçada que recobre uma úlcera, em geral grande, dando um aspecto de amora (PONTES et al., 2000; BORAKS, 1996). Muitas vezes há envolvimento periodontal e periapical, que pode causar esfoliação espontânea dos dentes, ou manifestações da doença após uma exodontia. (PAGLIARI et al., 1995; LIMONGELI et al., 1978).

Os aspectos radiográficos das lesões periodontais manifestam como alargamento do seu espaço, destruições ósseas, osso alveolar com alteração da lâmina dura e reabsorções das cristas interdentárias (CERRI et al., 1986; LASCALA, MOUSSALI, 1983).

As lesões ósseas ocorrem mais freqüentemente no tórax e nos membros vindo a seguir o crânio e a bacia (CERRI et al., 1986; DEL NEGRO, 1986).

As lesões são osteolíticas bem delimitadas com reação óssea circundante e mal discernível. Essa reação óssea circundante é bastante discreta, sendo percebida nas lesões um pouco maiores e em radiografias de boa qualidade. O tamanho das lesões varia de alguns milímetros a alguns centímetros (CERRI et al., 1986).

$\mathrm{Na}$ radiografia de tórax pósteroanterior mostra um infiltrado de piócitos no terço médio dos pulmões, significando sinais característicos da Paracoccidioidomicose. Já na Tuberculose mostra lesões nos ápices dos pulmões fazendo um diagnóstico diferencial.

No exame histopatológico pode revelar áreas de ulceração na superfície do epitélio de revestimento. O Paracoccidioides brasiliensis leva a uma resposta inflamatória granulomatosa caracterizada por macrófagos e células gigantes multinucleadas. Há áreas com necrose e com fibrose cicatricial. Leveduras grandes e dispersas são prontamente identificadas após a coloração com Grocott-Gomori mentenamina de prata ou com o método PAS. Os fungos mostram freqüentemente múltiplos 
brotos-filhos presos à célula-mãe por pontes citoplasmáticas, gerando uma aparência de leme de navio (NEVILLE et al., 1998).

O tratamento é essencialmente clinico através de sulfas. Atualmente as sulfas de ação lenta (cetoconazol e itraconazol) têm sido preferidas. A Anfotericina B ficou restrita aos casos de risco de vida ou casos muito graves. (PONTES et al., 2000; BISINELLI et al., 1998; BORAKS, 1996; CERRI et al., 1986; TOMMASI, 1989). Esta indicação reservada é devido à sua nefrotoxidade e às reações de hipersensibilidade (ORTEGA et al., 1986; BRUMMER et al., 1993).

Dentre os derivados imidazólicos, o itraconazol é atualmente a droga de escolha, já que tem vantagem de produzir remissão imediata dos sinais e sintomas, sem causar alterações no metabolismo endócrino (ORTEGA et al., 1986; BRUMMER et al., 1993).

\section{A Paracoccidioidomicose é a} única micose sistêmica sensível à ação das sulfas ou de sulfas associadas a outras drogas. As sulfonamidas, como a sulfadiazina e o sulfisoxazole, apresentam vantagens como o baixo custo e a relativa baixa toxicidade, mas são requeridos longos períodos para o tratamento, de até cinco anos, observando-se ainda significativa taxa de recorrência, em torno de $25 \%$ dos casos acompanhadas da resistência do fungo à droga (ORTEGA et al., 1986; BRUMMER et al., 1993).

Em um estudo realizado no Estado do Paraná, em pacientes que foram submetidos à terapia com sulfadiazina, após seis meses de tratamento, observou-se regressão total das lesões da mucosa bucal, do lábio e da laringe. (BISINELLI et al., 1998).

O objetivo deste trabalho foi realizar uma avaliação de casos em pacientes com diagnóstico de Paracoccidioiodomicose que manifestaram algum tipo de lesão bucal primaria durante os anos de 2005 a 2008, no município de Volta Redonda, localizado na região Sul - Fluminense (Estado do Rio de Janeiro), por ser área considerada favorável geograficamente para este tipo de doença e a importância do diagnóstico precoce pelo Cirurgião Dentista.

\section{MATERIAL E MÉTODO}

A metodologia aplicada foi por abordagem indutiva com procedimentos estatísticos e descritivos e a técnica utilizada foi a documental indireta, com utilização de prontuários dos pacientes atendidos nesse período. 
Realizou-se levantamento nos arquivos do Centro de Doenças Infecciosas da Secretaria Municipal de Saúde de Volta Redonda de pacientes com manifestação bucal em lesão primaria da Paracoccidioiodomicose, no período de 01 de janeiro de 2005 a 31 de outubro de 2008. (Ficha clínica 1).

Ficha 1 - Modelo da ficha 1 utilizada na coleta dos dados. "Paracococidioidomicose com Manifestação Bucal - Casuística em Volta Redonda nos Anos de 2005 a 2008"

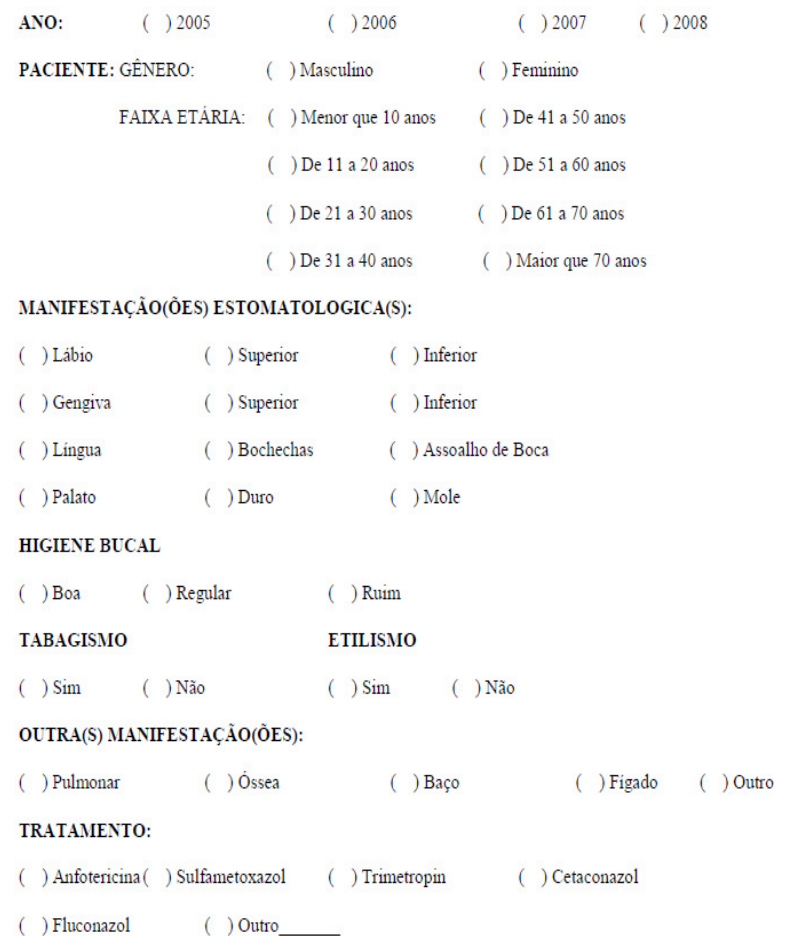

Esta pesquisa foi realizada de acordo com as normas de ética do Ministério da Saúde através da resolução $n^{\circ}$ 196/96, onde foram revisados 11 prontuários médicos. $\mathrm{O}$ método de diagnostico foi clinico, a partir dos dados semiológicos e clínicos, histopatológicos para as lesões bucais e radiográficos para as lesões pulmonares. O tratamento oferecido foi clinico através de prescrição de sulfas e o regime foi ambulatorial. Não se avaliou o prognóstico devido ao tratamento ser longo e alguns dos pacientes ainda estarem em tratamento.

\section{RESULTADOS}

Após análise dos prontuários, encontraram-se 11 (onze) onde a doença teve origem bucal. Destes, sete $(63,64 \%)$ eram do gênero masculino e quatro $(36,36 \%)$ feminino, dez $(90,90 \%)$ eram da raça branca e um $(9,10 \%)$ da raça negra, cinco $(45,45 \%)$ na faixa etária entre 41 e 50 anos, três $(27,27 \%)$ entre 51 e 60 anos, dois $(18,18 \%)$ entre 61 e 70 e um $(9,09 \%)$ acima de 70 anos.

Para as lesões bucais a língua foi o local preferido em seis $(54,54 \%)$, sendo que em um $(9,09 \%)$ estava associado aos lábios, seguido da gengiva com três $(27,27 \%)$ casos e somente nos lábios com dois $(18,18 \%)$ casos. Figuras 1 e 2. 


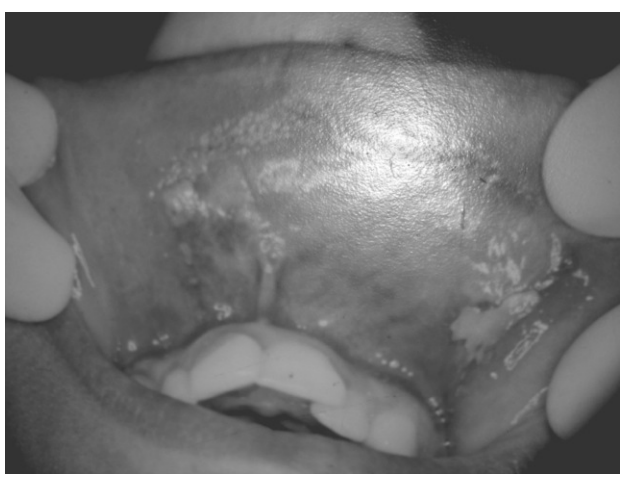

Fig.1- Paracoccidioidomicose labial superficial

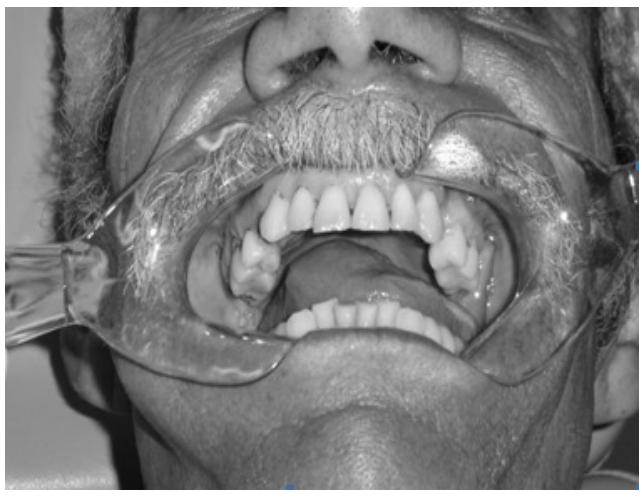

Fig. 2 - Paracoccidioidomicose profunda de língua

Histologicamente apresentam o

Paracoccidioides Brasiliensis, em meio a denso infiltrado inflamatório crônico, dentro e fora das células gigantes componentes de granuloma. As lesões apresentam área de ulceração de mucosa recoberta por material fibrinoleucocitário, contendo células gigantes, células epitelióides, infiltrado inflamatório mononuclear e o Paracoccidioides Brasiliensis. Figuras 3 e 4 .

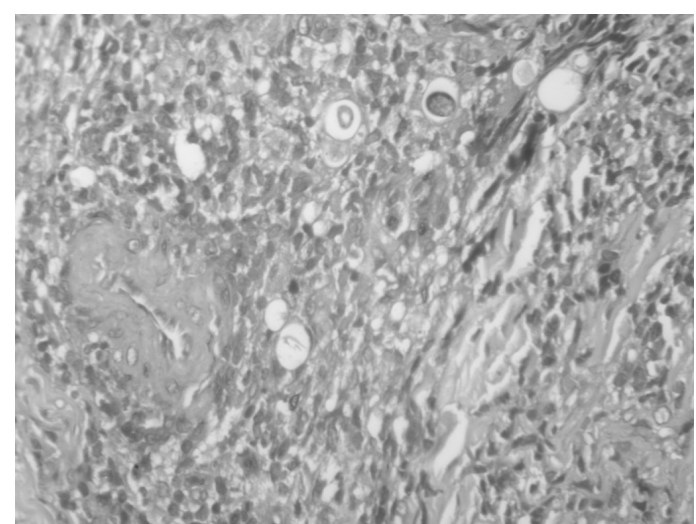

Fig. 3 - Paracoccidioides Brasiliensis, em meio a denso infiltrado inflamatório crônico

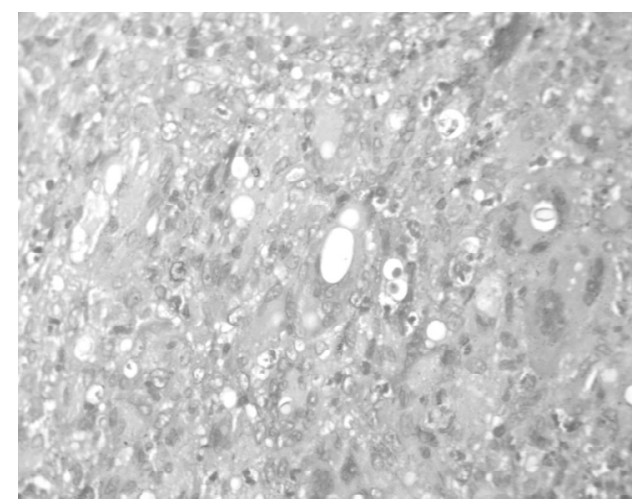

Fig. 4 - Fungos dentro e fora das células gigantes

Em relação à saúde e higiene bucal nenhum dos avaliados apresentou o mínimo aceitável, portanto todos (100 \%) dos avaliados apresentaram problemas com higiene bucal e, em conseqüência, tinham processos patológicos bucais e mucosa avermelhada e edemaciada. Dez $(90,90$ $\%$ dos 11 casos alegam serem tabagistas ou terem sido até o diagnostico confirmado, bem como sete $(63,63 \%)$ alegaram serem etilistas. 
Todos os $11(100 \%)$ casos apresentaram confirmação de lesão pulmonar, sendo que um $(9,09 \%)$ apresentou também complicação no baço.

O tratamento clínico medicamentoso em regime ambulatorial foi estabelecido de duas formas:

a) primeira escolha:

administrados dois comprimidos de Cetoconazol 200mg por dia por um período de seis meses, associado a Sulfametoxazol $400 \mathrm{mg}$ e Trimetropin $80 \mathrm{mg}$ também com dois comprimidos por dia/cada por um período de um ano. Oito $(72,72 \%)$ pacientes tiveram esta opção de tratamento.

$$
\text { b- segunda escolha: }
$$
administrados um comprimido de Fluconazol 150mg a cada sete dias por um período de seis meses, associado ao Sulfametoxazol $400 \mathrm{mg}$ e Trimetropin $80 \mathrm{mg}$ com dois comprimidos por dia por um período de um ano. Três $(27,27$ \%) pacientes tiveram esta opção.

Em dois meses as lesões bucais encontram-se em processo de cicatrização e em oito meses apresentam a remissão total dos sintomas. Tabelas 2 e 3.
Tabela 1 - Resultados da amostra: "Paracococidioidomicose com Manifestação Bucal - Casuística em Volta Redonda nos Anos de 2005 a 2008"

\begin{tabular}{|c|c|c|c|c|c|c|}
\hline PACIENTE & GÊNERO & COR & FAIXAETÁRIA & $\begin{array}{l}\text { MANIFESTAÇ̃̃ES } \\
\text { HISTOLÓGICAS }\end{array}$ & $\begin{array}{c}\text { OUTRAS } \\
\text { MANIFESTAÇÕES }\end{array}$ & MEDICAÇÃO \\
\hline 1 & M & B & 41 a 50 anos & Lesãoem gengiva & Pulmonar & $\begin{array}{l}\text { Sulfametoxazol } \\
\text { +trimetropin e } \\
\text { cetoconazol }\end{array}$ \\
\hline 2 & $\mathrm{~F}$ & $\mathrm{~N}$ & 41 a 50 anos & Lesãoem lábio inferior & Pulmonar & $\begin{array}{l}\text { Sulfametoxazol } \\
\text { +trimetropin e } \\
\text { cetoconazol }\end{array}$ \\
\hline 3 & M & B & 51 a 60 anos & Lesãoem língua & Pulmonar & $\begin{array}{l}\text { Sulfametoxazol } \\
\text { +trimetropin e } \\
\text { cetoconazol }\end{array}$ \\
\hline 4 & M & B & 61 a 70 anos & Lesãoem gengiva & Pulmonar & $\begin{array}{l}\text { Sulfametoxazol } \\
\text { + trimetropin e } \\
\text { cetoconazol }\end{array}$ \\
\hline 5 & M & B & 51 a 60 anos & Lesãoem língua e lábios & Pulmonar & $\begin{array}{l}\text { Sulfametoxazol } \\
\text { +trimetropin e } \\
\text { cetoconazol }\end{array}$ \\
\hline 6 & M & B & 61 a 70 anos & Lesãoem língua & Pulmonar & $\begin{array}{l}\text { Sulfametoxazol } \\
\text { +trimetropin e } \\
\text { fluconazol }\end{array}$ \\
\hline 7 & M & B & Mais de 70 anos & Lesãoem língua & Pulmonar & $\begin{array}{l}\text { Sulfametoxazol } \\
\text { +trimetropin e } \\
\text { fluconazol }\end{array}$ \\
\hline 8 & M & B & 51 a 60 anos & Lesãoem gengiva & Pulmonar & $\begin{array}{l}\text { Sulfametoxazol } \\
\text { +trimetropin e } \\
\text { cetoconazol }\end{array}$ \\
\hline 9 & $\mathrm{~F}$ & B & 41 a 50 anos & Lesãoem lingua & Pulmão e Baço & $\begin{array}{l}\text { Sulfametoxazol } \\
\text { +trimetropin e } \\
\text { cetoconazol }\end{array}$ \\
\hline 10 & $\mathrm{~F}$ & B & 41 a 50 anos & Lesãoem lábio inferior & Pulmonar & $\begin{array}{l}\text { Sulfametoxazol } \\
\text { +trimetropin e } \\
\text { fluconazol }\end{array}$ \\
\hline 11 & $\mathrm{~F}$ & B & 41 a 50 anos & Lesãoem língua & Pulmonar & $\begin{array}{l}\text { Sulfametoxazol } \\
\text { +trimetropin e } \\
\text { cetoconazol }\end{array}$ \\
\hline
\end{tabular}

\section{DISCUSSÃO}

O Paracoccidioides Brasiliensis, segundo Londero (1982), Cerri et al., (1986) e Cabral et al., (2000) é um fungo tem como habitat os vegetais e a terra fértil de vales, nas proximidades de grandes rios e vegetações abundantes ou extensas lavouras, com fatores geográficos de altitude entre 100 e 1.500 metros, matas tropicais e subtropicais e solos ácidos e fatores climáticos de temperatura entre $11 \mathrm{e}$ $30^{\circ} \mathrm{C}$, pluviometria entre 800 e 2.000 $\mathrm{mm}$ por ano. Segundo o Instituto Brasileiro de Geografia e Estatística IBGE (2000), estas características 
climáticas, pluviométricas e vegetativas assemelham-se com a região do Vale do Paraíba Sul Fluminense, em especial Volta Redonda, município onde foi realizado o presente estudo. Assim, embora seja uma cidade com perfil geoeconômico industrial, o município e a região possuem áreas rurais.

A transmissão deste fungo é feita por via inalatória, implicando em lesão primaria pulmonar, ou através da contaminação pela pele ou mucosas com solução de continuidade. A mucosa bucal de pacientes com má higiene é susceptível a lesões como gengivites e estomatites o que serve de porta de entrada para a instalação de uma lesão primaria bucal, principalmente para aqueles que contatam com vegetais contaminados, através da boca (LONDERO et al., 1982; CERRI et al., 1986; LASCALA, MOUSSALI, 1983).

Foram encontrados 11 (onze) prontuários de pacientes com a doença de origem bucal no período estudado entre 2005 a 2008.

Dos 11 prontuários, observou-se que sete $(63,64 \%)$ pacientes eram do gênero masculino, dados que corroboram com aqueles apresentados por Bisinelli (1998), em estudo realizado no estado do Paraná com 45 pacientes com a doença, destes 39 pacientes $(86,67 \%)$ pertenciam ao gênero masculino e seis $(13,33 \%)$ ao gênero feminino.

De acordo com Neville et al., (1998), a notável diferença pela predileção pelo gênero masculino em relação ao feminino, é explicada pelo efeito protetor do hormônio betaestradiol (hormônio feminino), que inibe a transformação da forma de leveduras dos microorganismos para a forma patogênica das hifas. Mesmo assim não se deve desconsiderar que o fungo tem como habitat o meio rural e que este trabalho é uma atividade mais a cargo do homem, o qual está conseqüentemente mais expondo ao fungo.

Dos casos avaliados, dez $(90,90 \%)$ deles eram em pacientes brancos e apenas um $(9,10 \%)$ em negro. Isto deve ser em decorrência da presença da melanina, a qual deve oferecer resistência ao fungo em sua penetração na mucosa bucal.

A faixa etária mais acometida está entre 41 e 50 anos, com cinco $(45,45 \%)$ casos. É o período produtivo em que o organismo começa a ter predominância do catabolismo com conseqüente queda da resistência orgânica. Não foram detectadas infecções em pacientes abaixo de 40 anos o que corrobora com a afirmativa acima. A redução da casuística acima de 
60 anos deve-se a saída do campo a partir desta idade.

Embora os autores descrevam a gengiva como a área mais afetada por lesão primária Cerri et al., (1986), Lascala, Moussali, (1983), neste trabalho a preferência foi para a língua com seis $(54,54 \%)$, sendo que em um $(9,09 \%)$ estava associado aos lábios, e a gengiva com três $(27,27 \%)$. As lesões iniciais têm como preferência marcante pela cavidade bucal, sendo esta uma das características.

Todos (100 \%) os pacientes avaliados apresentaram higiene bucal deficitária e alterações na mucosa, como eritema e edema, associados ao tabagismo em dez $(90,90 \%)$ casos e ao etilismo em sete $(63,63 \%)$. São pacientes rudes do campo e apresentam estas importantes características clínicas bucais.

Todos os $11(100 \%)$ casos apresentaram confirmação de lesão pulmonar, sendo que um $(9,09 \%)$ apresentou também complicação no baço. Segundo Ortega e Cols. (1996) o envolvimento pulmonar crônico está presente em cerca de $70 \%$ dos pacientes, os quais exibem tosse, dispnéia, febre, perda de peso e hemoptise (ORTEGA et al., 1996).

A confirmação diagnóstica das lesões bucais, em todos os casos, foi realizada através do exame clínico, com avaliação das características patognomônicas das lesões com granulações assemelhando-se a amoras, e histopatológicas, a partir de tecidos colhidos por biópsias incisionais, em sua maioria, onde o Paracoccidioides Brasiliensis aparece a meio de denso infiltrado inflamatório, dentro e fora das células gigantes componentes de granuloma.

A Paracoccidioidomicose é a única micose sistêmica sensível à ação das sulfas ou de sulfas associadas a outras drogas. As sulfonamidas, como a sulfadiazina e o sulfisoxazole, apresentam vantagens como o baixo custo e a relativa baixa toxicidade, mas são requeridos longos períodos para o tratamento, de até cinco anos, observando-se ainda significativa taxade recorrência, em torno de $25 \%$ dos casos acompanhadas da resistência do fungo à droga (ORTEGA et al., 1986; BRUMMER et al., 1993).

Dos pacientes avaliados todos foram tratados em regime ambulatorial com medicação antifúngica por um período de seis meses a um ano. A Anfotericina B em regime hospitalar não foi utilizada em nenhum dos casos, pela sua toxidade e às reações de hipersensibilidade Ortega et al., (1986), Brummer et al., (1993), sendo restrita 
aos casos de risco de vida ou casos muito graves. (PONTES et al., 2000; BISINELLI et al., 1998; BORAKS, 1996; CERRI et al., 1986; TOMMASI, 1989).

Alguns autores utilizam os derivados imidazólicos, o itraconazol é atualmente a droga de escolha, já que tem vantagem de produzir remissão imediata dos sinais e sintomas, sem causar alterações no metabolismo endócrino (ORTEGA et al., 1986; BRUMMER et al., 1993).

Mesmo após a alta os pacientes devem continuar sendo avaliados para evitar possível recorrência das manifestações clínicas.

\section{CONCLUSÕES}

A cidade de Volta Redonda no Vale do Paraíba Sul-Fluminense possui todas as características geográficas e climáticas favoráveis ao desenvolvimento do o Paracoccidioides

\section{Brasiliensis}

Devido à acentuada frequiência de lesões primarias bucais, o Cirurgião Dentista tem papel importante no diagnostico precoce da doença.

A preferência pelo gênero masculino pode ser justificada por estes estarem mais expostos aos fungos no campo e pelo feminino ser protegido pelo hormônio betaestradiol.

A predileção pela raça branca pode estar correlacionada à falta de melanina e conseqüente menor resistência tecidual.

A faixa etária mais acometida está entre 41 e 50 anos, com cinco $(45,45 \%)$ casos, período produtivo em que começa o catabolismo e queda da resistência orgânica.

As lesões iniciais têm como preferência marcante a cavidade bucal, estando associadas à higiene bucal deficitária, ao tabagismo e ao etilismo, acometendo mais a língua.

Todos os pacientes estudados possuíam lesão pulmonar, mostrando que nenhum deles foi diagnosticado precocemente.

O tratamento clínico ambulatorial foi realizado com Sulfametoxazol e Trimetropim associados ao Cetoconazol, em primeira escolha, ou ao Fluconazol.

\section{REFERÊNCIAS}

BISINELLI, J.; MARZOLLA, C.; FERREIRA, M.L.S.; TOLEDOFILHO; J.L.; PASTORI, C.M.; ZORZETTO, D.L.G. Manifestações estomatológicas da Paracoccidioidomicose (Paracoccidioides Brasiliensis ou Enfermidade de Lutz-splendoreAlmeida) Considerações gerais e apresentação de casos. Revista 
Brasileira de otorrinolaringologia,

Vol.67, nº8, Pág 683-687, set 2001.

BORAKS, S. Diagnóstico Bucal. São

Paulo: Artes médicas, 1996.

BRUMMER, E.; CASTANEDAM, E.; RESTREPO A.

Paracoccidioidomycosis. An update.

Rev Clin Microbiol 6:89-92 (1993).

CABRAL L.A.G et al. Emprego da imunofluorescência direta no diagnóstico da Paracoccidioidomicose. Revista odontológica da USP, vol 08, No1, Pág 27-32, jan/mar 1994.

DEL NEGRO, G.

Paracoccidioidomicose II - Tratamento: noções práticas. J. Pneumol., 12: (1): 57-60, 1986.

CABRAL, L.A.G. Estudos imunohistoquímico das espécies celulares componentes dos quadros histopatlógicos presentes nas lesões bucais da Paracoccidioidomicose crônica. São José dos Campos, 1995. Tese ( livre-docência ) - Faculdade de odontologia de São José dos Campos, UNESP.

CERRI, A.; GENOVESE, W.J.; BORDINI, P.J.; SAMARTINE, R. Paracoccidioidomicose Periodontal. Odontólogo Moderno, Vol.13, nº8, Pág 13-8, 1986.

IBGE - Instituto Brasileiro de Geografia e Estatística - IBGE. Censo demográfico 2000.

LASCALA, N.T.; MOUSSALI, N.H. Periodontia Clínica. Editora Artes Médicas. Brasil. 1983.

LIMONGELLI, W.A.; ROTHSTEIN, S.S.; SMITH, L.G.; CLARCK, M.S. Disseminated South American blastomycosis (paracoccidioidomycosis): report of case. J Oral Surg. 1978 Aug;36(8):62530.

LONDERO A.T.; SANTOS, W.; SILVA, L.A.; RAMOS, C.D.

Paracoccidioidomicose associada por droga imunosupressora em paciente com lupus eritematoso sistêmico. $\mathbf{J}$ Pneumol 13:224-227 (1987).

MORATO-FERNANDES, R.N.; BERALDO,P.S.S.; MASINI,M.; COSTA, P.H.C.

Paracoccidoiodomicose de localização intra-medular e cerebral. Arq. Neuropsiquiátrico (São Paulo) 49(2); Pág 192197, 1991.

NEVILLE, B.W. et al. Patologia oral e maxilofacial. Rio de Janeiro; Ed.

Guanabara Koogan, Pág 168-169, 1998.

ORTEGA, K.L; OLIVEIRA, P.T; MAGALHÃES, M.H.C.G; ARAÚJO, N.S; A citologia esfoliativa no diagnóstico da Paracoccidioidomicose: relato de caso. Revista de Pósgraduação GMD, Vol 03, N02, abr/mai/jun 1996.

PAGLIARI, A.V.; ARCE, G.A.T.; PIAZZETA, C.M. Aspectos clínicos das lesões de Paracoccidioidomicose na mucosa bucal. DENS, Curitiba, Vol 11, Pág 41-48.Editora da UFPR, 1995.

PONTES, F.S.C.; PONTES, H.A.R.; MOREIRA, C.R.; DANIN, G.A; PEREIRA, E.M.

Paracoccidioidomicose- aspectos gerais e relatos de caso. BCI- Revista Brasileira de cirurgia e implantodontia Vol.7 - no 28- out/nov/dez 2000.

TOMMASI, A.F. Diagnóstico em Patologia Bucal. 2 ed. São Paulo: Pancast, 1989. 